\title{
Clinical characterization of hospitalized COVID-19 patients during the second wave of pandemic in the district of Rohrbach, Upper Austria
}

\section{A single center retrospective study}

\author{
Klemens Rosenberger · Friedrich Pöschl · Svetlin Geschev · Kostja Steiner · Stefan Puig • Julia Röper-Kelmayr • \\ Karl J. Aichberger
}

Received: 3 May 2021 / Accepted: 25 September 2021 / Published online: 21 October 2021

(C) Springer-Verlag GmbH Austria, part of Springer Nature 2021

Summary During the peak of the second wave of the coronavirus disease 2019 (COVID-19) pandemic in November 2020, the district of Rohrbach, Upper Austria, was reported to have had the highest 7-day incidence of severe acute respiratory syndrome coronavirus-2 (SARS-CoV-2) positive cases worldwide. In this study, we present the clinical characteristics of COVID-19 cases during the second wave of the pandemic in patients admitted to the only primary care hospital in the district of Rohrbach between October 2020 and February 2021. In total, 260 patients were hospitalized with a mean age of 72 years and a mortality rate of $14.6 \%$ and 13 patients (5\%) were transferred to the intensive care unit (ICU). Critically ill patients $(22.7 \%)$ were of older age and often lived in retirement and nursing facilities as compared to mild or moderately ill patients. Patients with a severe disease course showed significantly longer hospitalization, a worse peripheral oxygen saturation on admission and significantly higher levels of C-reactive protein (CRP), procalcitonin (PCT), lactate dehydrogenase (LDH), troponin I and D-dimer as compared to mild or moderate COVID-19 cases. These laboratory parameters might help to identify COVID-19 patients with a se-

K. Rosenberger · F. Pöschl · S. Geschev · K. J. Aichberger $(\triangle)$ Department of Internal Medicine, Klinikum Rohrbach, Rohrbach, Austria

karl.aichberger@ooeg.at

\section{K. Steiner}

Department of Anaesthesiology and Intensive Care, Klinikum Rohrbach, Rohrbach, Austria

\section{S. Puig · J. Röper-Kelmayr}

Department of Radiology, Klinikum Rohrbach, Rohrbach, Austria vere disease course. In conclusion, we could show that older, frail individuals are the most vulnerable group affected by COVID-19. Whether this trend in hospitalized patients continues with the persistence of the pandemic, the emergence of novel virus mutations, and the availability of several different vaccines is presently unclear and remains to be determined.

Keywords SARS-CoV-2 · Mortality · Laboratory features · Comorbidities · ARDS

\section{Introduction}

An unexplained pneumonia outbreak in Wuhan, China in late 2019 was detected by the Chinese authorities as having been caused by a new type of B-coronavirus [1]. This virus was named as severe acute respiratory syndrome coronavirus 2 (SARSCoV-2), and the World Health Organization (WHO) officially termed the disease caused by SARS-CoV-2 as coronavirus disease 2019 (COVID-19) [2]. COVID19 rapidly spread from Wuhan to other cities in China and the entire world. By the end of February 2020, several European countries reported COVID-19 cases [3]. On 11 March the WHO officially declared the outbreak a pandemic, and governments across the world began implementing strategies to slow the infection spread, such as social distancing and complete lockdown [4]. Italy soon became the new emerging epicenter in Europe. In contrast to Italy and some other European countries, the first wave of the pandemic did not affect Austria as much as compared to the second wave.

During the first wave of the pandemic, the authorities reported 153 SARS-CoV-2 positive cases in the district of Rohrbach between March and May 2020 with 
only 11 patients being hospitalized, whereas during the second wave of the pandemic between October 2020 and February 2021, 4408 COVID-19 cases were reported in the district with 260 patients being admitted to our hospital.

COVID-19 is mainly transmitted through respiratory droplets and close contact. Asymptomatic infections are a major source of transmission [5-7]. The incubation period of SARS-CoV-2 ranges from 2-14 days after virus exposure [8]. The most common symptoms include fever, cough, shortness of breath, muscle ache, headache, sore throat, and loss of taste or smell [9].

In terms of laboratory findings, many patients have a decreased number of whites blood cells (WBC) and a decreased number of lymphocytes. In most patients, C-reactive protein (CRP) and lactate dehydrogenase (LDH) levels are elevated, but procalcitonin (PCT) levels are often normal [10].

The virus spreads and invades through the respiratory mucosa triggering an immune response and induces a cytokine-release syndrome, which has been described as cytokine storm in patients with a severe progression of the disease [11]. In patients with severe COVID-19 the development of acute respiratory distress syndrome (ARDS) is characteristic and associated with high mortality, approximately $40 \%$ of patients with ARDS do not survive [9].

Computed tomography (CT) imaging is of utmost importance for the diagnosis and prognosis of COVID19. The chest CT findings of COVID-19 may include ground-glass opacities (GGO), crazy-paving pattern, consolidation, and other findings of viral pneumonia [12-14]. Chest CT can be used to evaluate the severity of lung involvement. The CT findings are related to the time course and often show different imaging signs with progression [12].

In the early phase of the disease, there are multiple interstitial changes, especially in the peripheral portions of the lungs. In severe cases, lung consolidation can occur, but pleural effusions are rare [12].

In the present study, we describe the clinical and radiologic findings of COVID-19 patients admitted to our hospital during the second wave of the pandemic from October 2020 until February 2021, in which the district was reported to have had the highest 7-day incidence of SARS-CoV-2 positive cases worldwide (1475 cases/100,000 inhabitants, 17 November 2020) [15-17]. By 20 November 2020, 1196 SARS-CoV-2 positive individuals were reported to the authorities in the district (2.1\% of the population!) [18]. The 7-day incidence of SARS-CoV-2 positive cases was 736/100,000 inhabitants in Upper Austria and $543 / 100,000$ inhabitants all over Austria by that point of time.

\section{Methods}

\section{Setting}

This study was performed at the Klinikum Rohrbach, the only primary care teaching hospital located in the district of Rohrbach, Upper Austria. The hospital has 196 beds and provides basic medical and surgical care with a department for internal medicine, a department for surgery and for trauma surgery. The hospital also has a pediatric ward, a department for gynecology and obstetrics, a department of radiology and a department for anesthesiology and intensive care for an average of 11,000 inpatients (10,791 inpatients in 2019) and 50,000 outpatients (49,436 outpatients in 2019).

\section{Study design}

We conducted a retrospective data analysis of the clinical and demographic characteristics of all hospitalized COVID-19 cases during the second wave of pandemic admitted to our hospital between October 2020 and February 2021. The study was approved by the Institutional Review Committee and all data were collected retrospectively.

\section{Data collection}

The following data were collected from medical records: patient characteristics, such as age, gender, comorbidities (arterial hypertension, coronary heart disease, diabetes mellitus, chronic obstructive pulmonary disease), laboratory features (leukocytes, lymphocytes, CRP, PCT, LDH, hypersensitive troponin I, D-dimer), peripheral oxygen saturation on admission, and length of hospitalization. Once a suspected case was admitted to the hospital, a nucleic acid test (PCR, GeneXpert, Cepheid, Sunnyvale, CA, USA) was carried out immediately by a nasopharyngeal swab and analysis in our laboratory to confirm positivity for SARS-CoV-2. A native thin-section chest CT was performed on every COVID-19 patient on admission.

COVID-19 patients were clinically classified into three major categories: first, mild disease course in patients requiring no oxygen support, second, moderate disease course in patients requiring oxygen support, and, third, severe/critically ill patients with either fatal outcome or survival and requirement of high flow oxygen therapy (Airvo), continuous positive airway pressure (CPAP) ventilation or invasive ventilation. We assessed the qSOFA score (respiratory rate, altered mental status, systolic blood pressure) as well as peripheral oxygen saturation and heart rate to determine the degree of illness and to check for clinical deterioration. Patients received additional treatment with methylprednisolone, antibiotic therapy or antithrombotic prophylaxis with low-molecular weight 
heparin when clinically indicated. A subset of patients also received remdesivir.

\section{Statistical analysis}

Continuous variables are represented by median and quartile ranges (P25 and P75), categorical variables are represented by numbers (percentages).

Mann-Whitney U-test and Krustkal Wallis test were used for nonnormal distribution variables. The $\chi^{2}$ or Fisher's exact test were used to analyze classified variables.

Age, gender, length of hospitalization, comorbidities (arterial hypertension, coronary heart disease, diabetes mellitus, chronic obstructive pulmonary disease), and laboratory features (leukocytes, lymphocytes, CRP, PCT, LDH, troponin I, D-dimer) as well as peripheral oxygen saturation were considered as relevant parameters. $P$-values below 0.05 were considered statistically significant. All analyses were performed with the SPSS program (Version 27.0) (IBM Corporation, Chicago, IL, USA).

\section{CT image acquisition and image analysis}

All patients underwent non-contrast CT of the chest in supine position with breath-holding after inspiration (GE CT Revolution HD/GSI; GE Healthcare, Chicago, IL, USA). The following scan parameters were used: tube voltage of $120 \mathrm{kV}$ and dose modulation, reconstructed at $0.625 \mathrm{~mm}$ and $2.5 \mathrm{~mm}$ slice thickness, using both standard lung windows $(1300 \mathrm{HU}$ width; $-500 \mathrm{HU}$ center) and soft tissue window (360 HU width; $50 \mathrm{HU}$ center). Scan coverage was from the apex of the lungs to the level of bilateral adrenals. Two chest radiologists evaluated the images on a picture archiving and communication system (PACS) workstation. An artificial intelligence(AI)-based automatic quantification of ground-glass opacities and consolidation was analyzed retrospectively, using the software application prototype CT-Pneumonia Analysis based on a Syngo.via VB40B workstation (Siemens Healthineers, Erlangen, Germany) and the standard (soft tissue) reconstruction with $0.625 \mathrm{~mm}$ slice thickness. Based on 3D segmentations of lesions, lungs, and lobes, the algorithm quantified the extent of overall abnormalities and the presence of high-opacity ab- normalities, both globally and lobe-wise. The overall values used in this evaluation were: total opacity score: range between 0 and 20 . To obtain this score, the opacity scores of the five lung lobes are summed up. Percentage of opacity: this value represents the percentage of opacity for the whole lung. The opacity score (range: $0-4$ ) of each lung lobe is calculated as follows: $\leq 1 \%$ of opacity; score $=0$; $\leq 25 \%$ of opacity; score $=1 ; \leq 50 \%$ of opacity; score $=2 ; \leq 75 \%$ of opacity; score $=3 ;>75 \%$ of opacity; score $=4$. This score was calculated for each lobe. For the left/right lung, the opacity score is the sum of the respective lobes and for the total opacity score, all lobe values are summed up (CT Pneumonia Analysis-Handbook, Siemens, Erlangen, Germany; 2020).

\section{Results}

\section{Study population and patient characteristics}

During the second wave of the pandemic, 260 COVID19 patients were admitted to our hospital. The demographic and clinical characteristics of the patients are shown in Table 1. Patients had a mean age of 72 years (63-82 years interquartile range, IQR), and displayed a slight male predominance with $56.5 \%$. The most frequent medical comorbidities of hospitalized COVID19 patients were arterial hypertension (50.8\%), diabetes mellitus (14.6\%), coronary heart disease (10.4\%), and chronic obstructive pulmonary disease (COPD) (5.0\%) (Table 3). An AI-based automatic quantification of ground-glass opacities and consolidation in thin-section non-contrast chest CTs was performed on every patient on admission. One representative patient is shown (Fig. 1).

\section{Length of hospital stay}

The total length of hospitalization of COVID-19 patients was 6 days (3-11 days IQR) (Table 1). Patients with a severe/fatal disease course had a significantly longer length of hospital stay (11 days, 6-22 days IQR) as compared to patients with a mild (3 days, $2-5$ days IQR) or moderate disease course (7 days, 4-11 days IQR) (Table 1). In addition, severely/critically ill patients were significantly older (76 years, 69-83 years IQR), as compared to mild (64 years, 54-80 years IQR)

Table 1 Clinical and demographic characteristics and of hospitalized COVID-19 patients $(n=260)$

\begin{tabular}{|l|l|l|l|l|l|l|}
\hline & Total $(n=260)$ & Mild $(n=75)$ & Moderate $(n=126)$ & Severe/fatal $(n=59)$ & $p$-Value & Kruskal-Wallis H \\
\hline & Mean $(25-75 \%$ IQR) & Mean $(25-75 \%$ IQR) & Mean $(25-75 \%$ IQR) & Mean $(25-75 \%$ IQR) & & \\
\hline Age (years) (range) & $72(63-82)$ & $64(54-80)$ & $74(64-84)$ & $76(69-83)$ & $<0.001^{\star \star *}$ & 16.984 \\
\hline Female $(n)(\%)$ & $113(43.5 \%)$ & $37(49.3 \%)$ & $60(47.6 \%)$ & $16(27.1 \%)$ & - & - \\
\hline Male $(n)(\%)$ & $147(56.5 \%)$ & $38(50.7 \%)$ & $66(52.4 \%)$ & $43(72.9 \%)$ & - & - \\
\hline Length of hospitalization (days) (range) & $6(3-11)$ & $3(2-5)$ & $7(4-11)$ & $11(6-22)$ & $<0.001^{\star \star *}$ & 74.817 \\
\hline Mortality $(n)(\%)$ & $38(14.6 \%)$ & 0 & 0 & $38(64.4 \%)$ & - & - \\
\hline $\begin{array}{l}\text { IQR interquartile range } \\
{ }^{*} p<0.05,{ }^{\star * \star} p<0.001\end{array}$ & & & & & & \\
\hline
\end{tabular}




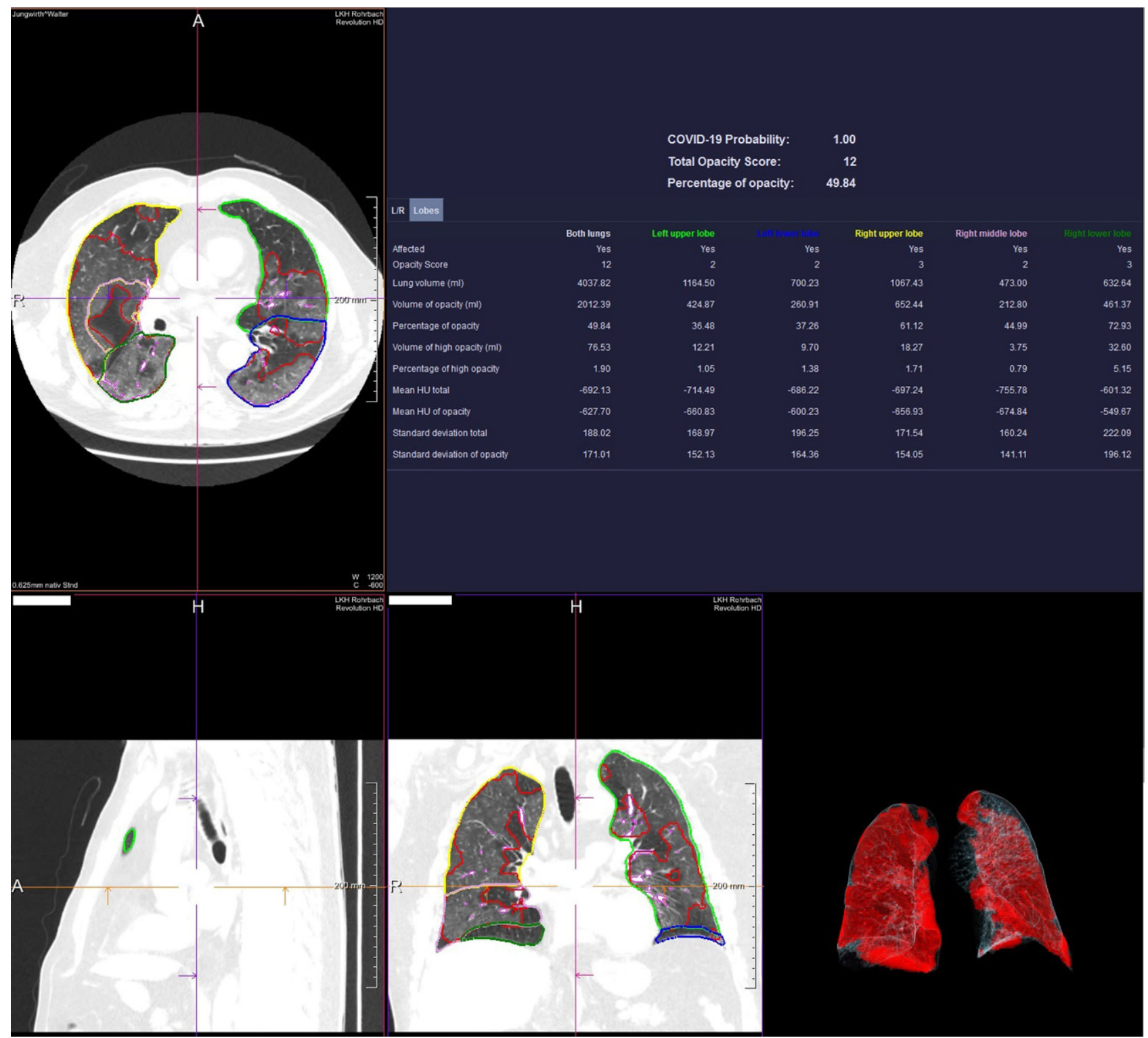

Fig. 1 Thin-section non-contrast chest CT of a representative COVID-19 patient with a severe disease course (patient 1). An Al-based automatic quantification of groundglass opacities and consolidation was analyzed using the software application prototype "CT-Pneumonia Analysis" based on a Syngo.via VB40B workstation (Siemens Healthineers, Er-

or moderately ill patients (74 years, 64-84 years IQR) (Table 1).

\section{Mortality}

The overall mortality rate of patients was $14.6 \%$ (38 of 260 patients) (Table 1), 59 patients $(22.7 \%)$ were classified as severely/critically ill, 13 patients (5\%) were transferred to the intensive care unit (ICU), 7 patients died on the ICU, 6 patients survived ICU and were transferred back to the normal medical ward and could be discharged later on. Of the COVID-19 patients with a critical condition 31 died on the normal langen, Germany) and the standard (soft tissue) reconstruction with $0.625 \mathrm{~mm}$ slice thickness. Based on 3D segmentations of lesions, lungs, and lobes, the algorithm quantified the extent of overall abnormalities and the presence of high-opacity abnormalities, both globally and lobe-wise

medical ward and were not transferred to ICU due to high age and/or severe underlying comorbidities, 126 patients $(48.5 \%)$ had a moderate disease course requiring additional oxygen support, whereas 75 patients $(28.8 \%)$ showed a mild disease course requiring no oxygen therapy (Table 1).

\section{Laboratory findings and comorbidities of hospitalized COVID-19 patients}

Patients with a severe/fatal disease course showed significantly higher numbers of leukocytes (mean 7.1 G/L, 5.3-9.7 G/L IQR) as compared to patients 
Table 2 Laboratory features of hospitalized COVID-19 patients $(n=260)$

\begin{tabular}{|c|c|c|c|c|c|c|}
\hline & Total $(n=260)$ & Mild $(n=75)$ & Moderate $(n=126)$ & Severe/fatal $(n=59)$ & $p$-Value & Kruskal-Wallis H \\
\hline & Mean $(25-75 \%$ IQR) & Mean (25-75\% IQR) & Mean $(25-75 \%$ IQR) & Mean $(25-75 \%$ IQR) & & \\
\hline Leucocytes (G/L) (4.0-10.0 G/L) & $6.2(4.8-8.2)$ & $6.2(4.6-7.7)$ & $6.1(4.5-8.5)$ & $7.1(5.3-9.7)$ & $0.028^{*}$ & 7.148 \\
\hline Lymphocytes (G/L) (0.6-5.0G/L) & $0.8(0.6-1.2)$ & $1.0(0.7-1.5)$ & $0.8(0.6-1.1)$ & $0.7(0.51-1.1)$ & $0.001^{\star \star \star}$ & 14.292 \\
\hline $\mathrm{CRP}(\mathrm{mg} / \mathrm{L})(0.0-5.0 \mathrm{mg} / \mathrm{L})$ & $\begin{array}{l}61.01 \\
(23.44-118.08)\end{array}$ & $24.25(6.6-62.3)$ & $\begin{array}{l}64.88 \\
(31.03-118.34)\end{array}$ & $\begin{array}{l}107.56 \\
(61.34-176.23)\end{array}$ & $<0.001^{\star \star \star}$ & 57.884 \\
\hline PCT (ng/mL) $(0.0-0.5 \mathrm{ng} / \mathrm{mL})$ & $0.07(0.04-0.19)$ & $0.05(0.03-0.08)$ & $0.07(0.04-0.15)$ & $0.22(0.07-0.67)$ & $<0.001^{\star \star \star}$ & 48.051 \\
\hline LDH (U/L) (125-220U/L) & $275(208-348)$ & $213(184-272)$ & $276(213-325)$ & $398(288-552)$ & $<0.001^{\star \star \star}$ & 57.971 \\
\hline D-dimer (mg/L) $(0.0-0.49 \mathrm{mg} / \mathrm{L})$ & $0.99(0.55-2.17)$ & $0.76(0.41-3.22)$ & $0.98(0.59-1.91)$ & $1.41(0.72-3.34)$ & $0.015^{\star}$ & 8.461 \\
\hline Troponin I-HS ( $\mu \mathrm{g} / \mathrm{L})(0.0-0.03 \mu \mathrm{g} / \mathrm{L})$ & $0.01(0-0.03)$ & $0.01(0.0-0.01)$ & $0.01(0.01-0.03)$ & $0.03(0.01-0.07)$ & $<0.001^{\star \star \star}$ & 61.004 \\
\hline Peripheral oxygen saturation (S02) (\%) & $92.8(90-95)$ & $95.0(93.6-96.7)$ & $92.0(89.2-94.0)$ & $90.0(86.0-93.2)$ & $<0.001^{\star \star \star}$ & 67.126 \\
\hline
\end{tabular}

Table 3 Comorbidities of hospitalized COVID-19 patients $(n=260)$

\begin{tabular}{|c|c|c|c|c|c|c|}
\hline & Total $(n=260)$ & Mild $(n=75)$ & Moderate $(n=126)$ & Severe/lethal $(n=59)$ & $p$-Value & $\chi^{2}$-test \\
\hline & Mean (25-75\% IQR) & Mean $(25-75 \%$ IQR) & Mean (25-75\% IQR) & Mean (25-75\% IQR) & & \\
\hline Comorbidities $(n)(\%)^{\mathrm{a}}$ & $158(60.8 \%)$ & $38(50.7 \%)$ & $83(65.1 \%)$ & $38(64.4 \%)$ & 0.104 & 4.520 \\
\hline Arterial hypertension $(n)(\%)$ & $132(50.8 \%)$ & $35(46.7 \%)$ & $67(53.35)$ & $30(50.8 \%)$ & 0.671 & 0.797 \\
\hline Diabetes mellitus ( $(n)(\%)$ & $38(14.6 \%)$ & $6(8 \%)$ & $21(16.7 \%)$ & $11(18.6 \%)$ & 0.148 & 3.822 \\
\hline Coronary heart disease $(n)(\%)$ & $27(10.4 \%)$ & $3(4 \%)$ & $18(14.3 \%)$ & $6(10.2 \%)$ & 0.060 & 5.344 \\
\hline COPD $(n)(\%)$ & $13(5.0 \%)$ & $2(2.7 \%)$ & $5(4 \%)$ & 6 (10.2\%) & 0.107 & 4.461 \\
\hline
\end{tabular}

with a mild (mean $6.2 \mathrm{G} / \mathrm{L}, 4.6-7.7 \mathrm{G} / \mathrm{L} \mathrm{IQR}$ ) or moderate disease course (mean $6.1 \mathrm{G} / \mathrm{L}, 4.5-8.5 \mathrm{G} / \mathrm{L} \mathrm{IQR}$ ) (Table 2). By contrast, patients with a severe/fatal disease course showed significantly lower numbers of lymphocytes (mean $0.7 \mathrm{G} / \mathrm{L}, 0.51-1.1 \mathrm{G} / \mathrm{L} \mathrm{IQR}$ ) as compared to patients with a mild (mean $1.0 \mathrm{G} / \mathrm{L}$, 0.7-1.5G/L IQR) or moderate disease course (mean $0.8 \mathrm{G} / \mathrm{L}, 0.6-1.1 \mathrm{G} / \mathrm{L}$ IQR) (Table 2). Patients with a severe/fatal disease course showed a significantly worse peripheral oxygen saturation on admission (mean 90.0\%, 86.0-93.2\% IQR) as compared to patients with a mild (mean 95.0\%, 93.6-96.7\% IQR) or moderate disease course (mean $92.0 \%, 89.2-94.0 \%$ IQR) (Table 2). In terms of laboratory findings on admission, patients with a severe/fatal disease course showed significantly higher levels of CRP, PCT, LDH, troponin I, and D-dimer, as compared to patients with a mild or moderate disease course (Table 2). We found no significant differences between the three COVID-19 patient groups regarding the frequency of medical comorbidities such as arterial hypertension, diabetes mellitus, coronary heart disease, or COPD. Overall, $60.8 \%$ of the patients had one or more of these comorbidities (Table 3).

\section{Discussion}

The district of Rohrbach, located in the northern part of Upper Austria, next to the border of Bavaria and the Czech Republic has a population of 56,545 people
(November 2020). Rohrbach was reported to be the area with the highest 7-day incidence of SARS-CoV-2positive cases worldwide during the second wave of the pandemic.

In this study we investigated all COVID-19 patients admitted to the Klinikum Rohrbach during the second wave of pandemic in a retrospective fashion. Overall, 260 patients were admitted, the mean age was 72 years, the overall mortality rate was $14.6 \%$ and 59 patients $(22.7 \%)$ displayed a severe disease course with acute respiratory distress syndrome (ARDS) (Table 1). COVID-19 appeared mostly as typical viral pneumonia on thin-section chest CT, which was performed on every patient on admission. The disease was mainly distributed around the subpleural areas, predominantly in the lower lobes. Ground-glass opacities (GGO) were the most common imaging manifestation (Fig. 1).

In contrast to previously published reports, the mortality rate of COVID-19 patients transferred to our hospital was rather high. A significant proportion of fatal cases were not transferred to ICU due to high age and severe underlying comorbidities. Many of these old and vulnerable patients were transferred to the hospital from retirement/nursing homes and care facilities, in which COVID-19 clusters emerged during the second wave of the pandemic.

In terms of laboratory findings, we were able to show that hospitalized COVID-19 patients with a severe disease course had significantly higher levels of 
CRP, PCT, LDH, troponin I, and D-dimer on admission as compared to mild or moderate cases. Similar findings have been published by Zhou et al., who postulated an 18-fold higher mortality for patients with $\mathrm{D}$-dimer values greater than $1 \mu \mathrm{g} / \mathrm{ml}$, as well as by Wang et al. who found significant higher values for leucocytes, D-dimer, LDH, GOT, GPT, and troponin I in ICU patients in comparison to non-ICU COVID19 patients [19, 20].

Thus, such laboratory parameters in addition to measurement of the peripheral oxygen saturation in consideration of comorbidities might be of help to identify COVID-19 patients at risk of having a severe disease course, which is in accordance with previously published reports [21, 22].

The reasons why the district of Rohrbach displayed the highest 7-day incidence of SARS-CoV-2 positive cases worldwide in November 2020 during the peak of the second wave of the pandemic still remain unclear. One explanation for the high number of COVID19 cases in this period of time might have been carelessness with respect to social distancing and a lack of adherence to lockdown sanctions.

Currently, there is still no specific drug for the treatment of patients with COVID-19. Some studies suggest positive therapeutic effects for remdesivir, when given at an early time point of infection. Overall, 19 of our patients $(7.3 \%)$ received remdesivir. In these patients, onset of clinical symptoms was shorter than 10 days $[23,24]$.

Our study has several limitations. First, this was a retrospective analysis and data were obtained from patient medical records, therefore, our data cannot be generalized or extrapolated. Second, the time frame of our observation period was rather short (4 months). At present (September 2021), Austria is approaching the fourth wave of the pandemic and the district of Rohrbach shows a 7-day incidence of SARSCoV-2 positive cases of around 160 cases/100,000 inhabitants. Vaccination against COVID-19 is currently ongoing and most of the inhabitants in retirement/ nursing homes in the district have already been vaccinated successfully or presumably died during the second wave of the pandemic. On the other hand, several new and more aggressive virus mutations have emerged.

In conclusion, our study showed that hospitalized COVID-19 patients during the second wave of the pandemic in the district of Rohrbach had a rather high mortality rate. Patients with a severe disease course were significantly older and had significantly higher CRP, PCT, LDH, troponin I, and D-dimer levels as compared to mild or moderate cases. Whether these trends in hospitalized patients continue with the persistence of the pandemic, the emergence of novel virus mutations, and the availability of several different vaccines is presently unclear and remains to be determined.
Acknowledgements We thank Renate Geretschläger, Florian Auberger, Günter Schwarz and Anna Hehenberger for skilful logistic and technical support.

\section{Declarations}

Conflict of interest K. Rosenberger, F. Pöschl, S. Geschev, K. Steiner, S. Puig, J. Röper-Kelmayr and K.J. Aichberger declare that they have no competing interests.

Ethical standards The study was approved by the Institutional Review Committee and all data were collected retrospectively.

\section{References}

1. Zhou P, Yang X-L, Wang X-G, Hu B, Zhang L, Zhang W, et al. A pneumonia outbreak associated with a new coronavirus of probable bat origin. Nature. 2020;579(7798):270-3.

2. WHO. Coronavirus disease 2019 (COVID-19) situation report 22. Geneva: World Health Organisation; 2020.

3. WHO. Coronavirus disease 2019 (COVID-19) situation report 40. Geneva: World Health Organisation; 2020.

4. WHO. Coronavirus disease 2019 (COVID-19) situation report51. Geneva: World Health Organisation; 2020.

5. Gandhi M, Yokoe DS, Havlir DV. Asymptomatic transmission, the Achilles' heel of current strategies to control Covid19. NEngl J Med. 2020;382(22):2158-60.

6. Meselson M. Droplets and aerosols in the transmission of SARS-CoV-2. NEnglJ Med. 2020;382(21):2063.

7. Morawska L, Cao J. Airborne transmission of SARSCoV-2: the world should face the reality. Environ Int. 2020;139:105730.

8. Centers for Disease Control and Prevention. Symptoms of coronavirus. 2020. https://www.cdc.gov/coronavirus/ 2019-ncov/symptoms-testing/symptoms.html. Accessed 22 Feb 2021.

9. Machhi J, Herskovitz J, Senan AM, Dutta D, Nath B, Oleynikov MD, et al. The natural history, pathobiology, and clinical manifestations of SARS-coV-2 infections. J Neuroimmune Pharmacol. 2020;15(3):359-86.

10. WuJ,LiuJ,ZhaoX, LiuC, WangW, WangD, etal. Clinicalcharacteristics of imported cases of Coronavirus disease 2019 (COVID-19) in Jiangsu province: a multicenter descriptive study. Clin Infect Dis. 2020;71(15):706-12.

11. Sun X, Wang T, Cai D, Hu Z, Chen J, Liao H, et al. Cytokine storm intervention in the early stages of COVID-19 pneumonia. Cytokine Growth Factor Rev. 2020;53:38-42.

12. Guan CS, Wei LG, Xie RM, Lv ZB, Yan S, Zhang ZX, et al. CT findings of COVID-19 in follow-up: comparison between progression and recovery. Diagn Interv Radiol. 2020;26(4):301-7.

13. Han R, Huang L, Jiang H, Dong J, Peng H, Zhang D. Early clinical and CT manifestations of Coronavirus disease 2019 (COVID-19) pneumonia. AJR Am J Roentgenol. 2020;215(2):338-43.

14. Li Y, Xia L. Coronavirus disease 2019 (COVID-19): role of chest CT in diagnosis and management. AJR Am J Roentgenol. 2020;214(6):1280-6.

15. Rohrhofer M. Mühlviertler Bezirk Rohrbach: Leben im Corona-Hotspot. 2020. https://www.derstandard. at/story/2000121972336/muehlviertler-bezirk-rohrbachleben-im-corona-hotspot2020. Accessed 26 Nov 2020.

16. Hofer E, Christandl J. Als das Virus die Stadt verließ: Weltweiter Corona-Hotspot lag im Mühlviertel. 2020. https://kurier.at/politik/inland/als-das-virus-die-stadtverliess/4011205712020. Accessed 6 Dec 2020. 
17. Gahleitner M. Corona-Neuinfektionen: Unrühmlicher Spitzenplatz für Bezirk Rohrbach. 2020. https://www.tips. at/nachrichten/rohrbach/land-leute/521159-coronaneuinfektionen-unruehmlicher-spitzenplatz-fuer-bezirkrohrbach2020. Accessed 17 Nov 2020.

18. Rohrbach Bezirkshauptmannschaft. 2020.

19. Zhou F, Yu T, Du R, Fan G, Liu Y, Liu Z, et al. Clinical course and risk factors for mortality of adult inpatients with COVID-19 in Wuhan, China: a retrospective cohort study. Lancet. 2020;395(10229):1054-62.

20. Wang D, Hu B, Hu C, Zhu F, Liu X, Zhang J, et al. Clinical characteristics of 138 hospitalized patients with 2019 novel Coronavirus-infected pneumonia in Wuhan, China. JAMA. 2020;323(11):1061-9.

21. Nakamura S, Kanemasa Y, Atsuta Y, Fujiwara S, Tanaka M, Fukushima K, et al. Characteristics and outcomes of coronavirus disease 2019 (COVID-19) patients with cancer: a single-center retrospective observational study in Tokyo, Japan. IntJClin Oncol. 2021;26(3):485-93.
22. Wei Y, Zeng W, Huang X, Li J, Qiu X, Li H, et al. Clinical characteristics of 276 hospitalized patients with coronavirus disease 2019 in Zengdu District, Hubei Province: a singlecenter descriptive study. BMCInfect Dis. 2020;20(1):549.

23. Frediansyah A, Nainu F, Dhama K, Mudatsir M, Harapan H. Remdesivir and its antiviral activity against COVID19: a systematic review. Clin Epidemiol Glob Health. 2021;9:123-7.

24. Feldt T, Karagiannidis C, Mager S, Mikolajewska A, Uhrig A, Witzke O, et al. Wie ist die aktuelle Datenlage zur Behandlung von COVID-19 mit Remdesivir? 2021. https://www. rki.de/DE/Content/InfAZ/N/Neuartiges_Coronavirus/ COVRIIN_Dok/Erfahrungen_Umgang_Erkrankten.pdf?_ blob=publicationFile2021. Accessed 11 Jan 2021.

Publisher's Note Springer Nature remains neutral with regard to jurisdictional claims in published maps and institutional affiliations. 